\title{
The combined use of weather radar and geographic information system techniques for flood forecasting
}

\author{
E. Baltas \\ AUTH, Agricultural School, Department of Hydraulics, Soil Science and Agricultural Engineering, 54006 Thessaloniki, \\ Greece
}

Received: 10 September 2006 - Revised: 20 November 2006 - Accepted: 27 January 2007 - Published: 26 April 2007

\begin{abstract}
A distributed rainfall-runoff model capable for real time flood forecasting utilizing highly spatial and time resolution data was developed. The study region is located under the WSR-74 S-band $100 \mathrm{~km}$ radar umbrella and is equipped with a number of rain gauge recording stations, a permanent installation for flow measurement and a stage recorder. The entire basin was digitized to $2 \times 2 \mathrm{~km}^{2}$ grid squares by implying GIS techniques. A series of rainfall events recorded producing floods were analyzed and processed. The linear channel parameter assigned to each gridsquare is based on its location measured by the centroid of the grid square along the channel network. The estimation of the hill-slope and the stream velocity are calculated based on the Geographic Information System (GIS) procedures.
\end{abstract}

\section{Introduction}

Flood flow forecasting for a catchment due to rainfall requires a quantitative estimate of precipitation. This can be accomplished using several methods including rain gauges, weather radar or a combination of rain gauges and weather radar. The traditional approach to flood forecasting is to use rainfall input estimated from a number of rain gauges driving a lumped parameter hydrological model. In rainfallrunoff transformation, lumped linear system models have been found to work, as well as, more physically based models (Plate et al., 1988). O'Connell et al. (1986) have shown the utility of the semi-distributed models for on-line forecasting of high resolution radar measurements. A number of mathematical models have been proposed by different authors to face the problem of flood forecasting (Baltas, 1998). In most of these models, combinations of linear conceptual elements are used to simplify the representation of hydro-

Correspondence to: E. Baltas

(baltas@agro.auth.gr) logical processes. Hydrologists have always been interested in the effects of rainfall uncertainties on the accuracy and reliability of the estimation of catchment-scale hydrological variables (runoff peak discharge, volume, etc.). The recent advent of new technologies such as weather radar, satellites, Geographic Information Systems and high-speed computer workstations, provides new opportunities for improved hydrological forecasting. This led to the development of distributed models for flood forecasting (Baltas and Mimikou, 1999). In more complex approaches, models for rainfall prediction were also applied. Together with the development of more feasible strategies, they can assist the operation of human-made structures and flood warning systems. This new technology led to the development of models for flood forecasting and of more complex models incorporating cloud physics linked to mesoscale dynamical models to provide rainfall forecasts for many hours ahead (Baltas, 1998). Furthermore, the lumped nature of the model, gives an intrinsic weakness to the model to represent extremely uneven spatial rainfall distributions, making it necessary to work in smaller sub-basins in such cases (Yang et al., 2000). Measurement accuracy continues to be a problem, but have not prevented self-correcting hydrological models from being developed for operational use.

In this paper, a procedure is suggested for the real-time quantitative radar rainfall measurements used for flood simulation and real-time flood forecasting. The model represents the basin by grid squares of $2 \times 2 \mathrm{~km}^{2}$ in area. Rain excess in each grid square is shifted linearly at the outlet of the basin. This model is an extension of the most common approach that is the division of a basin into sub-basins and channel segments and then the use of lumped rainfall-runoff models for the sub-basins and the combination of their responses after routing through the appropriate channel segments. Flood-flow forecasting in this area is very important because the region suffers from frequent and hazardous flash floods, causing damage and operational problems to

Published by Copernicus GmbH on behalf of the European Geosciences Union. 
Table 1. The radar technical characteristics.

\begin{tabular}{ll}
\hline Type & WSR-74 S-band \\
Wavelength & $10 \mathrm{~cm}$ \\
Dish Diameter & $12 \mathrm{ft}$ \\
Resolution of recorded data & 1 or $2 \mathrm{~km}$ (selectable) \\
Beamwidth & 2 degrees (maximum) \\
Size PPI display & 12 inches (diameter) \\
Size RHI display & $12 \times 12$ inches (rectangular) \\
A-indicator display & $4 \times 5$ inches (rectangular) \\
Sequence of elevation angles & $-1-+60$ degrees \\
\hline
\end{tabular}

the downstream multipurpose reservoirs and agricultural and residential areas.

\section{Study area and data processing}

Flood phenomena in Greece usually are caused by intense rainstorms, whereas snowmelt is not a dominant factor in flood genesis. Most intense rainstorms are produced by the passage of depressions possibly accompanied by cold fronts (and rarely by warm fronts) approaching from $\mathrm{W}, \mathrm{SW}$ or NW (Baltas and Mimikou, 1999). A convectional weather type (characterized by a cold upper air mass that produces dynamic instability) (Baltas, 1998a) is also responsible for many intense storms, especially in the summer period. The basin used in this study is the Pyli basin, located in the Thessaly water district under the $100 \mathrm{~km}$ radar umbrella with drainage area $135 \mathrm{~km}^{2}$ (Fig. 1). The plain is traversed by Pinios river whose total catchment area is $10500 \mathrm{~km}^{2}$, while the agricultural area is about $4000 \mathrm{~km}^{2}$. The orography of Pindos mountain range plays an important role in rainfall and runoff regimes in Greece. Thus, the mean annual rainfall exceeds $1800 \mathrm{~mm}$ in the mountainous areas of western Greece whereas in eastern regions of the country may be as low as $300 \mathrm{~mm}$ (Baltas, 1998b). This does not mean that extreme floods are uncommon in the relatively dry eastern part of Greece. Deforestation and urbanisation play an important role to flood genesis. They are responsible for the increasing severity and destructive power of floods. Deforestation, also related to soil erosion, is a major problem in Greece. It is noted that the percentage of the areas covered by forest today is $18 \%$, while at the beginning of the nineteenth century it was more than $40 \%$ (Baltas and Mimikou, 1999). Deforestation was caused mainly from human activities such as fires, illegal land reclamation, pasturing, etc. The topography varies from narrow gorges to wide flood plains. Its vegetation varies from grasslands to dense forest, while the climate is temperate and humid with substantial seasonal variation in temperature. Frequent and rapid changes in weather are caused by frontal air mass activity, resulting in frequent flash floods. Regarding the ground truth monitoring, the basin is equipped with a number of hydrometeorological recording stations, as well as, stage recording stations along with permanent installations for flow measurement at selected locations in the river reach. Historical storm and flood events have been recorded and archived by the Public Power Corporation (PPC), the Ministry of Agriculture and the Ministry of Environment, Physical Planning and Public Works. These consist of hourly precipitation data from rain gauges and the corresponding streamflow rates at the outlet of the basin. Streamflow is estimated by using the hourly water level readings and the appropriate water level-discharge rating curves calibrated at the site. The Thiessen polygon methodology was used in order to derive the meteorological mean values over the catchment.

The radar involved in the study is a WSR-74 S-band weather radar located in a military base in the town of Larissa in Central Greece. From this radar site, a significant part of central and northern Greece is covered including the Pyli basin. A number of storm events included the ones that created flash floods, recorded every ten to thirty minutes, were analyzed and processed. A variety of factors causing systematic random and range dependent errors are encountered in these estimates. After the suppression of the ground clutter map, the subtraction of the errors due to anomalous propagation, beam refraction and losses, interference of signal from other rain targets was carried out for rainfall estimation (Krajewski, 1987). The Marshall-Palmer Z-R exponential relationship was applied for the conversion of the radar reflectivity data to rainfall estimates, considering $R$ as the rainfall on the ground and $Z$ reflectivity factor measured by the radar. In the field of radar-rain gauge adjustment the Brandes method was developed for the need to merge radar and rain gauge rainfall information and thus to improve radar rainfall estimates, trying to explain quantitatively any discrepancies between the rainfall amounts indicated by the two different measurement techniques (Brandes, 1974).

A number of storm events creating flash floods were analyzed and processed. All these events belong to the same type of precipitation considered as cyclonic with moderate intensity, long duration and widespread rainfall distribution. Three dimensional polar coordinate maps of equivalent reflectivity factor $Z$ in dBZ values are recorded every ten to thirty minutes by the WSR-74 S-band radar. The most important radar technical characteristics are shown in Table 1.

\section{Radar rainfall estimation}

The power received by the radar antenna from a rainfall target is integrated in time and space to eliminate the characteristic fluctuation of the radar signal. The average received power can be expressed as:

$$
P_{r}=C K Z / r^{2}
$$




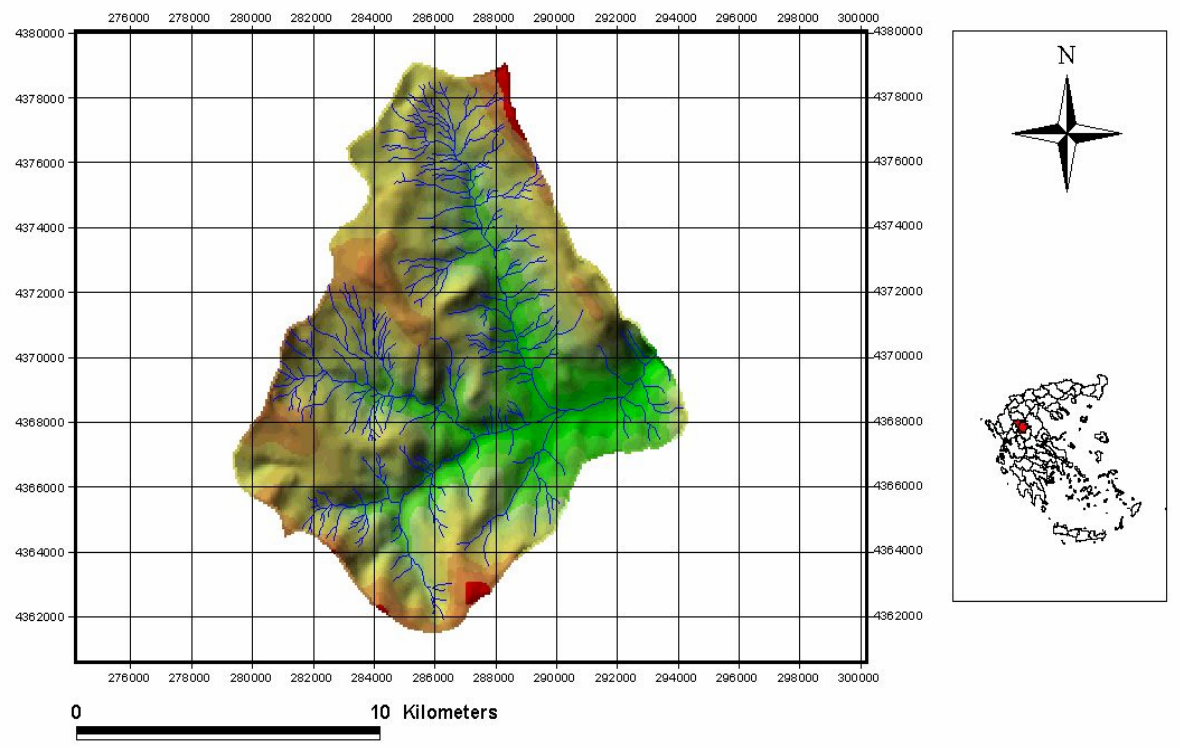

Fig. 1. The Pyli basin.

where $C$ is a constant depending on the parameters of the radar system and $K$ is the attenuation constant. To obtain rain rate $R$, the reflectivity factor $Z$ is used in a $Z-R$ relationship of the form:

$R=a Z^{b}$

where $a$ and $b$ are two parameters. Both $Z$ and $R$ are functions of the raindrop size distribution:

$Z=\int_{0}^{\infty} N(D) D^{6} d D$

and

$R=\frac{\pi}{6} \int_{0}^{\infty} D^{3} N(D) w(D) d D$

where $D$ is drop diameter, $N(D)$ is raindrop size distribution and $w(D)$ is a function describing terminal velocity of raindrops. It is obvious from the analysis of Eqs. (3) and (4) that even for the simple, two-parameter exponential distribution:

$N(D)=N_{o} \exp (-\Lambda D)$

there is no unique relationship between $Z$ and $R$, therefore, rainfall estimation based on (2) will lead to errors. However, there is a variety of factors that can cause systematic, random and range dependent errors. A short list of factors affecting the accuracy of rainfall derived from radar measurements is shown below (Zawadski, 1984):

1. High variability of raindrop size distribution which determines the $Z-R$ relationship.

2. Changes in the vertical reflectivity profile.
3. Bright band effects caused by scattering of radar waves by ice particles present in the higher levels of some clouds.

4. False echo caused by anomalous propagation of radar waves.

5. Miscalibration of radar electronic instruments.

6. Erroneous measurements of the $P_{r}$.

7. Atmospheric processes between the level of the measurement cell and the ground.

\section{Processing of the weather radar data}

The raw radar data measured in polar co-ordinates in $\mathrm{dBZ}$ values are converted to Cartesian ones with grid dimensions $2 \times 2 \mathrm{~km}^{2}$. A large area under the radar umbrella suffers from ground echoes which is a fact prohibiting radar utilization for such areas. Since the WSR-74 S-band radar system is not "dopplerized", a ground clutter map measured during a cloudless sky in the optimum elevation, was utilized for the detection and suppression of the ground clutter from radar rainfall fields. After the averaging of radar reflectivity data in whole hours, the well known Marshall-Palmer $Z-R$ exponential relationship was applied for the conversion of the radar reflectivity data to rainfall estimates. The reason that the Marshall-Palmer relation was used is that all the events analyzed and processed are found after implying (off-line) least square methods to have $a$ ranging from 170 to 215 and $b$ from 1.2 to 1.6 relatively close to the Marshall-Palmer coefficients, where $a=200$ and $b=1.6$. $Z-R$ relationships are 


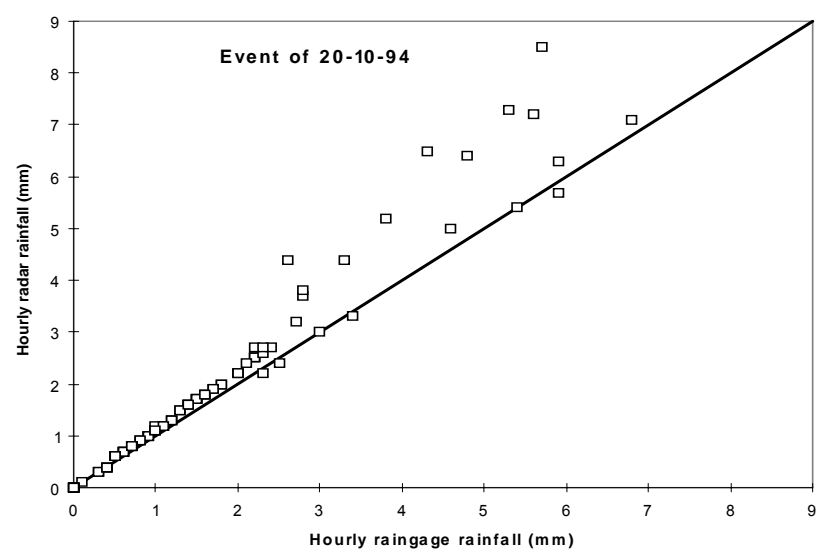

Fig. 2. One-to-one correspondence between the mean rain gauge hourly and the mean radar hourly rainfall.

derived for the various locations of the rain gauge stations considering $R$ as the rainfall on the ground and $Z$ reflectivity factor measured by the radar.

The next step is the application of the influence function method (Krajewski, 1987). It is applied to solve the problem of outliers detection resulting from errors due to abnormal beam refraction and losses, some type of anomalous propagation and interference of signal from other than rain targets. The influence function method is based on analysis of spatial correlation in the field and applied to radar rainfall fields. The reason that this method was selected is that applied to the same type of precipitation considered as cyclonic, doesn't require any external information or calibration, as well as any knowledge of distribution of the data and can be fully automated and used in an on line mode.

\section{Radar rain gauge combination}

In order to cope with biases in radar rainfall estimate the joint use of radar and rain gauge data has been recommended (Doviak and Zrnic, 1984). Joint use of radar and rain gauge data aims at the utilization of the high point accuracy of the latter and the wide area coverage of the former.

An approach based on the Brandes method was developed for the need to merge radar and rain gauge rainfall information and thus, to improve radar rainfall estimates, trying to explain quantitatively any discrepancies between the rainfall amounts indicated by the two different measurement techniques. The main characteristics of the Brandes method (Brandes, 1974), based on $G / R$ ratio, is the comparison between radar and rain gauge measurements in order to achieve adjustment factors which are used to force radar estimates to agree with rain gauge observed values. In this method an adjustment factor derived by using the rain gauges located in a selected range around that point is assigned to each grid point. The adjustment factor $F_{1}$ at each grid point is a weighted mean of the $N$ rain gauge observations:

$$
F_{1}=\sum_{i=1}^{N} W_{T i} G_{i} /\left(\sum_{i=1}^{N} W_{T i}\right)
$$

where $W_{T i}$ is a weight function depending on the range from the rain gauge to the grid point $r$ given by:

$W_{T i}=\exp \left(-r^{2} / E P\right)$

where $E P$ is a filtering parameter considered constant.

In all storm events after the processing of the radar data, the comparison of the hourly estimated radar rainfall to the hourly measured rain gauge rainfall are relatively close, as representatively shown in Fig. 2 for the event occurred on 20-10-94.

\section{Form of the distributed grid model}

The basin is divided into $N$ grid squares. Each grid square is assumed to transform excess rain by a transfer function. The transfer function is defined by a series of combination of linear channels and a linear reservoir. The linear channel parameter is a function of the location of the grid square. The linear reservoir parameter is assumed to be the same for all grid squares. The time of first rise at the observed hydrograph decides the time of start of rainfall excess in each grid square.

The contribution of a grid square at time $t$ is given by the equation:

$$
Q_{t}=\sum_{j=t_{r}-\left(t_{C, g}+\lambda\right)}^{i=t-\left(t_{C, g}+\lambda\right)} R_{j, g} U_{i-j+1}
$$

Combining the contribution of all grid squares for which $i>0$ gives

$$
Q_{t}=\sum_{g=1}^{N} \sum_{j=t_{r}-\left(t_{C, g}+\lambda\right)}^{i=t-\left(t_{C, g}+\lambda\right)} R_{j, g} U_{i-j+1}
$$

where $N$ is the number of grid squares, $Q_{t}$ is the computed discharge at time $t$ and $U_{t}$ are discrete values computed assuming the response function to be a linear reservoir.

Rainfall excess is computed using two infiltration models. These models consider the rain prior to the time $t_{r}-\left(t_{c, g}+\lambda\right)$ as initial loss. In the first scheme, the abstraction rate is considered constant. In the second scheme, the Philip's equation is used to define the infiltration process and the parameters are assumed to be the same for each grid square.

The Philip's equation is given by:

$$
F=S t^{0.5}+A t
$$

or

$f=\frac{1}{2} S t^{-0.5}+A$ 
where $S$ is called sorptivity, a function of initial and surface water contents of the soil and soil-water diffusivity, $A$ is a parameter depending upon soil properties, $F$ is the accumulated infiltered water as time $t$ elapses and $f$ is the infiltration rate.

The resulting equations incorporating the infiltration schemes are:

$Q_{t}=\sum_{g=1}^{N} \sum_{j=t_{r}-\left(t_{C, g}+\lambda\right)}^{i=t-\left(t_{C, g}+\lambda\right)}\left(P_{j, g}-\Phi\right) U_{i-j+1}$

and

$Q_{t}=\sum_{g=1}^{N} \sum_{j=t_{r}-\left(t_{C, g}+\lambda\right)}^{i=t-\left(t_{C, g}+\lambda\right)}\left(P_{j, g}-S\left(t^{0.5}-(t-1)^{0.5}\right)-A\right) U_{i-j+1}$

where $P_{j, g}$ is the precipitation at time $j$ on grid $g, \Phi$ is the average abstraction rate, $S$ is the sorptivity and $A$ is a constant parameter for Philip's method.

\section{Estimation of the parameters}

The parameters of the model represented by Eq. (12), are $\lambda$, $t_{c, g}, K$ and $F$ and those represented by Eq. (13), are $\lambda, t_{c, g}$, $K, S$ and $A$. The parameters which are determined off-line are $\lambda, t_{c, g}$ and $A$. The parameter $\lambda$ denotes the initial lag and is a time delay parameter taken the same for all grids (Fattorelli and Scaletta, 1991). The parameter $t_{c, g}$ is a time parameter assigned to each grid square on the basis of its location measured by the centroid of the grid square along the channel network. The $t_{c, g}$ values were derived based on historical events through two different ways, first by using various pairs of velocities off-channel and in channel network and secondly by implying the Nash hydrograph to the historical events.

First, the linear channel parameter assigned to each grid square is based on its location measured by the centroid of the grid square along the channel network. The time to the outlet of the basin is given as follows:

$t=S_{h} / V_{h}+S_{s} / V_{s}$

where $S_{h}$ the distance from the center of the grid to the stream, $S_{s}$ the distance from the stream for each grid to the exit of the basin, $V_{h}$ the hill slope velocity and $V_{s}$ the stream velocity.

The estimation of the hill slope and the stream velocity are calculated based on GIS procedures. These values were calibrated based on the unit hydrograph which was derived based on historical events.

The later helped through the calculation of the first and second moment arms of the excess rainfall and direct runoff hydrograph to the estimation of the Nash parameters $n$ and $K$ for each flood event. Table 2 shows the derived values of $n$
Table 2. The derived values of $n$ and $K$ for each flood event.

\begin{tabular}{cccc}
\hline No. & Date of event & Parameter $n$ & Parameter $K$ \\
\hline 1 & $14-01-91$ & 2 & 6.4069 \\
2 & $09-02-91$ & 5 & 3.16169 \\
3 & $06-04-91$ & 5 & 5.385 \\
4 & $10-05-91$ & 2 & 6.96 \\
5 & $17-11-91$ & 3 & 6.30421 \\
6 & $18-04-92$ & 2 & 6.18 \\
\hline
\end{tabular}

and $K$ for each flood event. A mean value of both parameters was used for the construction of the response formation $h(t)$ with time.

The third parameter estimated off-line, is the parameter of Philip's infiltration scheme A, a function of the soil properties and equals to the $2 / 3$ of hydraulic conductivity term (Singh, 1992).

The parameters which are to be estimated on line have been identified as $F$ and $K$ in the first infiltration scheme and $S$ and $K$ in the second scheme. The on line estimation of the parameter is performed based on the minimization of an objective function. This objective function is the summation of the square differences between the observed and computed flows.

Each grid square operates as a linear reservoir model, a one parameter $(K)$ model. The form of the instantaneous unit hydrograph of a linear reservoir is the following:

$h(t)=\frac{1}{K} e^{-t / K}$

Convoluting any input with the system response given in Eq. (15) and implying a conversion unit factor, will result in the corresponding discharge in $\mathrm{m}^{3} / \mathrm{s}$. The response function will be hourly integrated for the estimation of the discrete values $U_{t}$.

The criteria that are used in order to optimize the initial values of the parameters concern the minimization of an objective function, defined as the summation of the squares of differences between the observed and estimated discharge each time step. The objective function which is minimized during the optimization of the parameters is the following:

Objective function $=\left((Q \operatorname{obj}(i)-Q \operatorname{comp}(i))^{2} W T(i)\right)^{0.5} / N^{0.5}$

where $Q \operatorname{obj}(i)=$ the observed discharge in time $i, Q \operatorname{comp}(i)$ $=$ the computed discharge in time $i, W T(i)=$ the weight coefficient for ordinate $i$ and $N=$ the total number of ordinates.

For the estimation of the discharge in each time step, the number of ordinates taken into account for the minimization of the objective function is limited and is given based on the weight coefficient defined by the following equation:

$\frac{j^{2}}{(N-1)^{2}}$ 


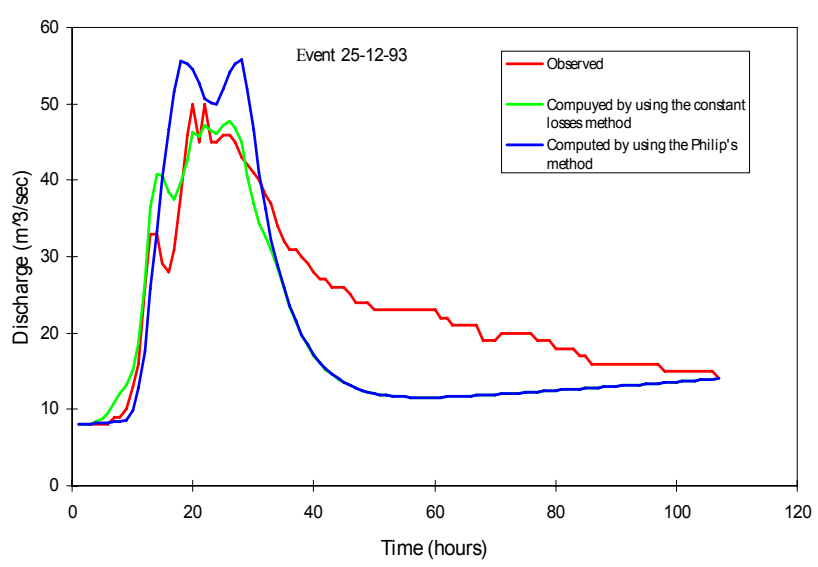

Fig. 3. The simulated hydrographs by using the constant losses and the Philip's method versus the observed.

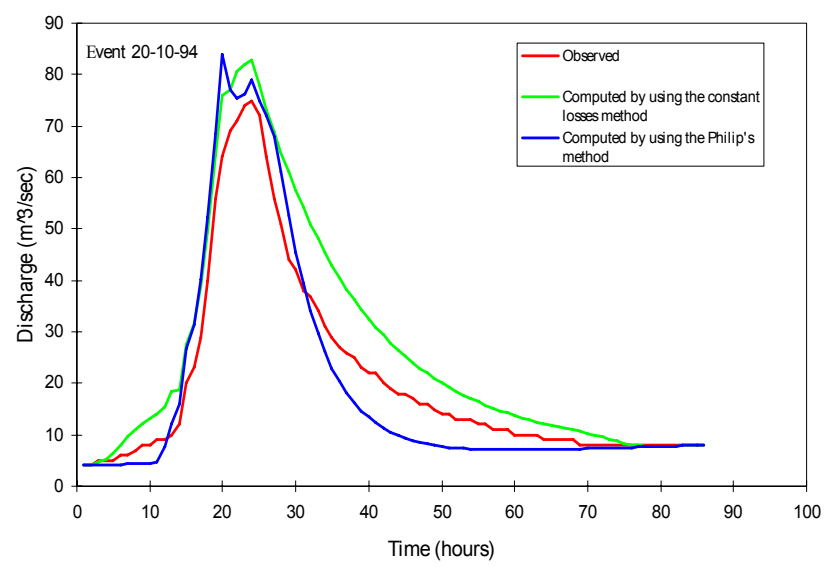

Fig. 4. The simulated hydrographs by using the constant losses and the Philip's method versus the observed.

where $j=$ the number of time steps $\Delta T$ from the beginning of the period used for the calculation of the parameter.

\section{Results}

Model runs were performed in two phases. First in the simulation phase and second in the forecasting phase. Equations (12) and (13) were used to simulate the hydrographs. Representative results for the simulation phase using both the mean constant losses method and the Philip's one, as well for the two of the events examined, are shown in Fig. 3 and 4. In these figures one can observe the relatively good agreement between the observed and computed hydrographs. The stochastic error component computing the time series of error between the observed and computed values from the time of first rise of the hydrograph until the time of forecast, was employed. The time series were analyzed to obtain the correlation structure for fitting an autoregressive model. The AR(1)

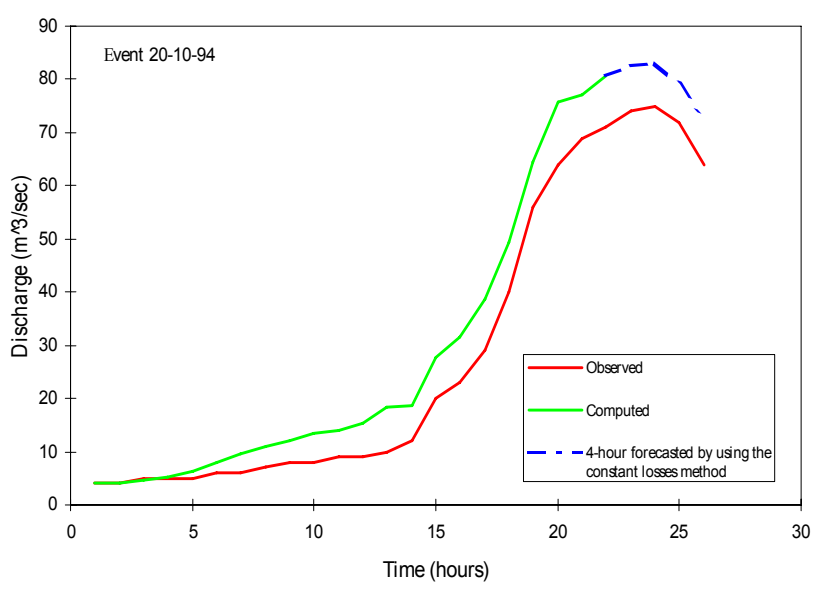

Fig. 5. Four-hour flood forecasting using the constant loss method.

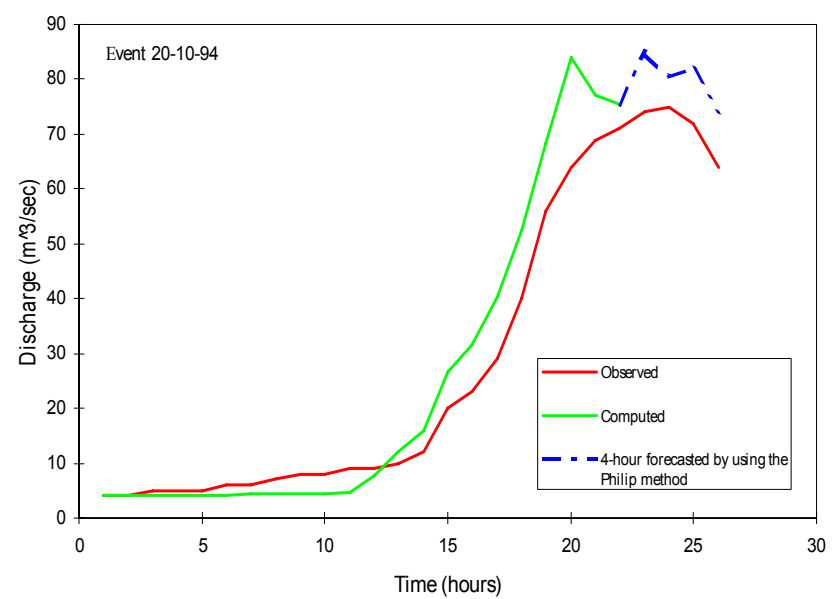

Fig. 6. Four-hour flood forecasting using the Philip's method.

and AR(2) models were selected. The parameters were determined and checked for stationarity. If the parameters of the AR(2) model do not satisfy the stationarity condition, then the AR(1) model is used. The standard deviation of the residual series is also computed for the evaluation of the AR(1) and AR(2) models and for the selection of one of these. The parameters of the autoregressive model are now used to compute the forecast errors beyond the time of forecast. These errors are added to the computed forecast discharges. Forecasts were carried out by setting lead time equal to two and four-hour ahead, covering the rising limb until the peak had passed with results of acceptable accuracy. Representative results for the four-hour flood forecast using the mean constant losses and the Philip's method are shown in Fig. 5 and 6 respectively. 


\section{Conclusions}

The main conclusions derived from this research work are concentrated on the following:

1. A series of storm events creating flash floods were analyzed and processed. The entire basin was divided in grid squares of dimensions $2 \times 2 \mathrm{~km}^{2}$. An adaptive grid-square-based flood-forecasting model capable of utilizing rainfall information on each grid square using weather radar, is proposed. Two different ways (one using GIS methods and one by implying the Nash hydrograph) were used to define the time delay of each grid square to the outlet of the basin. The model is able to formulate simulated hydrographs with relatively close agreement to the observed ones, as well as acceptable forecasts with a maximum lead time of $4 \mathrm{~h}$. The $4 \mathrm{~h}$ time period is little less than the time delay between the occurrence of effective rainfall and the production of its main effects at the Pyli basin.

2. Two infiltration schemes have been used to compute the rainfall excess. It emerges from the study that the grid model using the average abstraction rate as the infiltration model computes better forecasts for the majority of the flood events analyzed.

3. The updating of the parameters was performed in real time, every time step minimizing an objective function. This objective function is a summation of the square differences between the observed and computed discharge values. The autoregressive model was implied for the simulation of the error term.

Edited by: S. C. Michaelides and E. Amitai Reviewed by: anonymous referees

\section{References}

Baltas, E. and Mimikou, M.: A Grid-based rainfall-runoff model for flood forecasting, Proc. III International Symposium on Hydrological Applications of Weather Radars, Sao Paolo Brazil, August 1995 .

Baltas, E.: Distributed modeling of river basin runoff in Greece, Radar Hydrology for Real Time Flood Forecasting, An Advanced Study Cource, University of Bristol, UK, 24 June-3 July, 1998, 1998a.

Baltas, E.: Radar based for real-time flood forecasting in the Pinios river basin, in: International RIPARIUS Workshop on "Flood Risk Assessment, Communication, Present Practice and Future Needs", Brussels, 27-28 October, 1998, pp. 99-111, 1998b.

Baltas, E. and Mimikou, M.: Comparison of rainfall - runoff models based on spatial rainfall, Hydrotechnika, Hellenic Hydrotechniki Union, 9, 27-40, 1999.

Brandes, E. A.: Optimising rainfall estimates with the aid of radar, J. Appl. Meteor., 14, 1339-1345, 1974.

Doviak, R. J. and Zrnic, D. S.: Doppler radar and weather observations, Academic Press, Orlando, Florida, 1984.

Fattorelli, S. and Scaletta M.: Real-time flood forecasting models intercomparison, Advances in Radar Hydrology, International Workshop, Lisbon, 11-13 November, 1991.

Krajewski, W. F.: Radar rainfall data quality control by the influence function method, Water Resour. Res., 23(5), 837-844, 1987.

O' Connell, P. E., Brunsdon, G. P., Reed, D. W., and Whitehead, P. G.: Case studies in real-time hydrological forecasting from the UK, in: River flow modelling and forecasting, edited by: Kraijinhoff, D. A. and Moll, J. R., Reidel, Dordrecht, pp. 195-238, 1986.

Plate, E. J., Ihringer, J., and Lutz, W.: Operational models for flood calculation, J. Hydrol., 100, 489-506, 1989.

Singh, P. V.: Elementary Hydrology, Prentice Hall, Englewood Cliffs, N.S., 1992.

Zawadzki, I.: Factors affecting the precision of radar measurements of rain, Preprints of the 22nd Conference on Radar Meteorology, American Meteorological Society, Boston, Massachusetts, 1984.

Yang, D., Herath, S., and Musiake K.: Comparison of different distributed hydrological models for characterization of catchment spatial variability, Hydrol. Processes, 14, 403-416, 2000. 